\title{
La durabilité des sablières. Approche méthodologique dans sa perspective territoriale, les sablières Lafarge Granulats Ouest (LGO) en Bretagne
}

\section{Anaïs Guerin-Chapel}

\section{OpenEdition}

\section{Journals}

Édition électronique

URL : http://journals.openedition.org/cdg/2123

DOI : $10.4000 / \mathrm{cdg} .2123$

ISSN : 2107-7266

\section{Éditeur}

UMR 245 - CESSMA

\section{Référence électronique}

Anaïs Guerin-Chapel, «La durabilité des sablières. Approche méthodologique dans sa perspective territoriale, les sablières Lafarge Granulats Ouest (LGO) en Bretagne », Carnets de géographes [En ligne], 1 | 2010, mis en ligne le 01 octobre 2010, consulté le 24 septembre 2020. URL : http:// journals.openedition.org/cdg/2123; DOI : https://doi.org/10.4000/cdg.2123

Ce document a été généré automatiquement le 24 septembre 2020

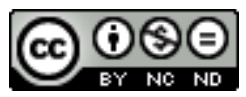

La revue Carnets de géographes est mise à disposition selon les termes de la Licence Creative Commons Attribution - Pas d'Utilisation Commerciale - Pas de Modification 4.0 International. 


\title{
La durabilité des sablières. Approche méthodologique dans sa perspective territoriale, les sablières Lafarge Granulats Ouest (LGO) en Bretagne
}

\author{
Anaïs Guerin-Chapel
}

\section{Problématique}

1 L'émergence du concept de "développement durable » et sa déclinaison progressive dans les politiques nationales et locales façonne les activités humaines avec de plus en plus d'impacts sur les modes de consommation. Ainsi, les industriels se trouvent confrontés à une double logique directement issue de cette évolution conceptuelle : l'obligation d'assurer leur rôle de moteur économique et la nécessité de se «mettre au vert " face à l'inquiétude toujours croissante de la population autour des risques industriels. Ainsi s'agit-il, pour le chercheur, d'analyser le développement durable, non pas uniquement comme une entité conceptuelle, mais bien dans sa relation physique et matérielle entre l'entreprise et ses parties prenantes. Nous intéressant à une industrie particulière - celle de l'extraction de sable - et après avoir étudié les aspects contextuels généraux associés à la prise en compte du développement durable au niveau de l'entreprise, nous nous questionnons sur la nature du prisme à adopter pour analyser cette relation.

- Selon quelles perspectives une sablière peut-elle être durable?

- Comment mesurer cette durabilité ?

2 En effet, plusieurs éléments propres à ce type d'industrie nous conduisent à envisager le développement durable d'une sablière, non comme une réponse presque pavlovienne de l'entreprise à son contexte, mais comme la mise en œuvre de procédures, d'actions $\hat{R}$ 
formant un ensemble plus complexe Ŕ s'inscrivant dans de multiples dimensions ou aspects. S'interroger sur la durabilité d'une industrie d'extraction suppose de ne pas s'arrêter à la seule question de la ressource. Celle-ci étant non durable, le premier point d'achoppement de la réflexion surgit rapidement. La durabilité de l'activité d'extraction de sable, prise dans son ensemble est, de manière endogène, nulle et non avenue.

- Mais est-ce à dire que ce type d'industrie ne peut s'inscrire dans la notion de durabilité ?

Il nous semble plus fécond d'envisager cette mise en tension de notions $\mathrm{R}$ de durabilité d'une industrie et de non durabilité de la ressource qu'elle exploite $R$ paradoxales, au regard non plus d'elle-même, mais à d'autres échelles. Pour qui, pourquoi et comment une sablière peut être durable?

Nous postulons que la durabilité d'une sablière peut être appréhendable, au regard des différentes échelles qui la composent. Ce faisant, nous engageons une réflexion prenant en compte la complexité du réel. Nous considérons qu'une sablière se compose d'un emboîtement de quatre échelles spatiales : du grain de sable au gisement elle est plus largement établie sur un site et au sein d'un territoire. Une sablière alimente une filière économique et modèle un territoire. À la suite, nous nous permettons un glissement sémantique, du concept de développement durable à la notion de durabilité (Da Cunha, 2003), qui nous semble rendre possible une analyse à un "grain plus fin » de la réalité d'une activité économique. Il s'agit de chercher à comprendre comment une industrie dans sa recherche de durabilité, peut "façonner» et s'inscrire dans un territoire (hypothèse 1). La seconde hypothèse nous conduit. à intégrer les dimensions physiques (liées à la notion de différenciation quantitative), organisationnelles et sociales (liées à la notion de différenciation qualitative, c'est-à-dire en rapport avec la valeur sociale que les acteurs accordent aux espaces), dans l'appréhension du territoire "mouvant" d'une sablière (Stock dans Levy et Lussault, 2003). La mise en exergue de la durabilité d'une activité d'extraction de sable doit donc prendre en compte cette pluralité d'aspects du territoire, qu'elle façonne, mais dans lequel, dans le même temps, elle s'inscrit. La troisième hypothèse sous-tend que la notion de durabilité intègre une perspective dynamique potentielle, d'une part car le changement (paysager, d'usage, etc.) peut être durable; d'autre part, car la durabilité s'applique de manière variable selon ces échelles, et enfin, car la durabilité " générale » d'un site est essentiellement liée à l'acceptation sociale de cet espace physique. De cet ensemble d'hypothèses découle notre problématique. Modéliser la durabilité d'une activité d'extraction, c'est définir une méthodologie d'appréhension de la variabilité d'incarnation de cette durabilité.

\section{Méthode de recherche}

5 Nous avons travaillé à la définition de critères d'étude, d'indicateurs, à travers une double approche : territoriale et liée à la notion de durabilité. Le terrain a fait l'objet de sorties sur les sites, de rencontres informelles, d'entretiens, de participations à des réunions, etc., afin de recueillir trois sortes de données :

- Les données existantes,

- Les données récoltées/compilées,

- Les données créées 
Les données récoltées ont été d'ordre quantitatif et qualitatif. Les premières sont relatives au fonctionnement des sites d'extraction et de leurs répercussions sur leur environnement. Au- delà de cette validation quantitative, il s'agit aujourd'hui pour les industriels, confrontés à une problématique d'ancrage territorial, de mettre en place une méthodologie d'approche qualitative. La mise en place de notre méthodologie se veut multidisciplinaire, alliant regard environnemental, économique, politique et social. $\mathrm{Au}$ final, notre recherche est établie sur une approche pluridimensionnelle du territoire : administrative, physique, économique et sociale. Cette méthodologie a été testée sur des cas concrets de sablières en Bretagne, les sites appartenant à Lafarge Granulats Ouest. L'aboutissement de notre travail d'analyse en grille d'évaluation a également fait l'objet d'un test sur les sites d'étude pour permettre de valider l'efficacité de l'outil.

\section{Aire géographique de l'étude}

$7 \quad$ Le choix de l'aire géographique de l'étude s'est porté sur les sites de sablières implantés en milieu rural, en réponse à une problématique d'actualité de "rejet » de nouveaux projets dans la région Bretagne. Le développement de la contestation sociale peut s'expliquer par un changement du monde rural. L'espace rural est actuellement - et ce depuis les années soixante-dix - de moins en moins modelé par la société paysanne et par l'agriculture. Au sein de cet espace rural, de nouvelles pratiques se développent et entraînent un « renouveau » du rural. Différentes fonctions sont tour à tour mises en place : elles peuvent être résidentielles, récréatives - «les usages récréatifs de l'espace agricole sont une réalité sociale (...) » (Le Caro Y., 2007) -, d'accueil d'activités renouvelées, écologiques et culturo-éducatives.

\section{Fiche informative}

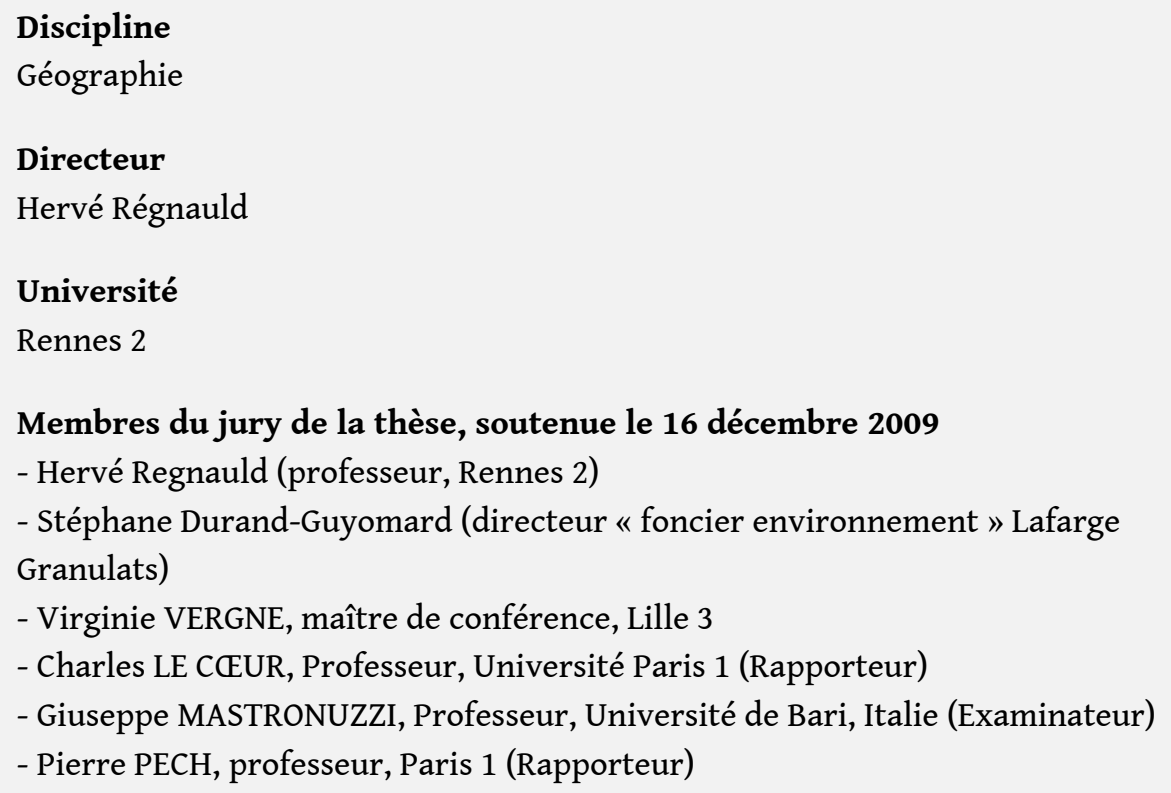


Situation professionnelle actuelle

chercheur associée au laboratoire $\operatorname{COSTEL}^{1}$ UMR 6554 LETG CNRS IFR/CAREN ${ }^{2}$, Université de Rennes 2

\section{Contact}

anais.guerinchapel[at]gmail.com

NOTES

1. COSTEL : Climat, Occupation du Sol par TELedection

2. CAREN : Centre Armoricain de Recherche en ENvironnement

INDEX

Thèmes : Carnets de soutenances 